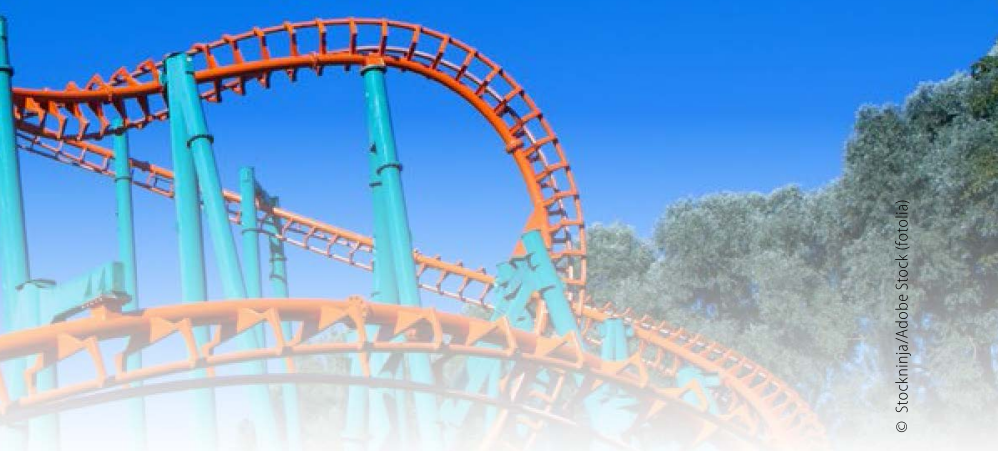

\title{
Achterbahnfahrt mit Schreck und Jubel
}

Als Diabetologe erlebt man eine Achterbahn der Gefühle. Höhepunkte waren die Publikationen der DCCT/EDIC- und der UKPDS-Daten. Der DCCT hat bei Typ-1-Diabetes (T1D) gezeigt, dass die normnahe Blutzucker-(BZ-)Einstellung mittelfristig mikround langfristig auch makrovaskuläre Komplikationen reduzieren kann. Ähnliche Ergebnisse ergab die UKPDS und ihr Follow-up bei neu entdecktem Typ-2-Diabetes (T2D). Die Folgerung: „Auf zur BZ-Senkung um jeden Preis!“ Dann kamen die kardiovaskulären (KV) Outcome-Studien bei T2D: ACCORD (2008), ADVANCE (2008) und VADT (2009) bei älteren Patienten, z. T. mit KV Erkrankungen. Diese ergaben, dass die strenge BZ-Einstellung nicht immer vorteilhaft und Unterzucker zu vermeiden ist. Nun hieß es: „BZ senken ja, aber bitte NICHT um jeden Preis!“

Zum gefühlten Tiefpunkt trug v. a. die Geschichte der Glitazone bei. Gestartet als Antidiabetika mit Gefäßschutz wurden 2007 Daten publiziert, die ein erhöhtes Herzinfarktrisiko mit Rosiglitazon suggerierten, sodass die Zulassung für Rosiglitazonpräparate 2010 zurückgezogen wurde. Zwar erwiesen sich die KV Daten später als nicht korrekt, der Todesstoß war aber gesetzt. Als Reaktion verschärfte sowohl die US- als auch die europäische $\mathrm{Zu}$ lassungsbehörde (FDA 2008, EMA 2010) die Auflagen für neue Antidiabetika, die seither ihre KV Sicherheit beweisen müssen.

Die ORIGIN-Studie ergab dann, dass Insulin glargin bei Personen mit gestörter Glukosetoleranz, neu entdecktem T2D oder kurzer Diabetesdauer zwar das KV und Krebsrisiko nicht steigert [1], aber auch, dass frühe Insulinisierung keine KV Vorteile hat.

Für Aufatmen sorgte auch die SAVOR-TIMI-53-Studie, die für den DPP-4-Hemmer Saxagliptin bei T2D plus KV Erkrankungen (KVE) oder hohem KV Risiko ebenfalls KV Neutralität zeigte [2]. Mit Saxagliptin kam es aber zu vermehrten Hospitalisationen wegen Herzinsuffizienz, ein Grund zur Sorge und Anlass zur Frage nach einem Klasseneffekt. Die Studie TECOS gab hier Entwarnung: Sitagliptin erhöhte bei T2D und KVE weder KV Endpunkte, noch Herzinsuffizienz-Hospitalisationen [3]. Unter Alogliptin war dekompensierte Herzschwäche dann auch nicht häufiger [4].

Befreiend wirkte die EMPA-REG-Outcome-Studie: Der SGLT2-Hemmer Empagliflozin reduziert bei T2D und hohem KV Risiko den primären Endpunkt (KV Mortalität, nicht tödlicher Schlaganfall und Herzinfarkt) mit der Hazard Ratio (HR) von 0,86. Zudem wurden KV und Gesamtmortalität (HR, 0,62; 0,68) sowie die Hospitalisationsrate wegen Herzschwäche (HR, 0,65) signifikant reduziert [5]. Ähnliches zeigte kürzlich die CAN-

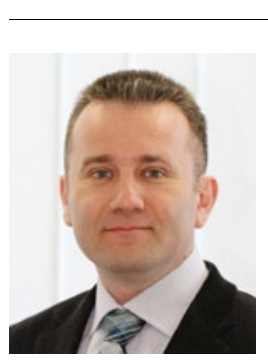

PD Dr. med. Ovidiu Alin Stirban

Facharzt für Innere Medizin, Endokrinologie und Diabetologie

Diabetologische Schwerpunktpraxis,Sana

Arztpraxen Remscheid GmbH

Alleestraße 105-107, 42853 Remscheid

ovidiualin.stirban@sana.de

VAS-Studie: Canagliflozin senkte vs. Placebo bei T2D plus hohem KV-Risiko den gleichen primären kombinierten Endpunkt wie in EMPA REG deutlich (HR 0,86), auch wenn Gesamt- und KV Mortalität separat nicht signifikant reduziert wurden [6]. Die Herzschwäche-Hospitalisationen nahmen auch ab (HR, 0,67), Amputationen (HR, 1,97) und Frakturen (HR, 1,26) aber zu. Für SGLT-2-Hemmer profiliert sich also ein Klasseneffekt bzgl. der KV Endpunkte, wenngleich sie verschieden stark beeinflusst werden. Daten zu Dapagliflozin stehen noch aus.

Weiter ging's mit dem GLP-1-Analogon Liraglutid beim selben kombinierten Endpunkt. Es reduzierte ihn bei T2D plus hohem KV Risiko (HR 0,87) sowie einzeln betrachtet die KV (HR, 0,78) und die Gesamtmortalität (HR, 0,85) [7]. Auch das einmal wöchentliche Semaglutid konnte KV Endpunkte reduzieren [8], für Lixisenatid wurde nur eine KV Neutralität belegt [9]. Ein Klasseneffekt ist für GLP-1-Analoga also nicht anzunehmen.

Im Gegensatz dazu häufen sich Daten zu negativen KV Effekten durch Sulfonylharnstoffe (SH). Da in ACCORD, ADVANCE und VADT viele Patienten SH nahmen, könnte dies einen Teil der negativen KV Effekte und der häufigen Hypoglykämien erklären.

Es verdichtet sich, dass weniger die Stärke der Glukosesenkung kardiovaskulär entscheidend ist, als vielmehr WOMIT dies geschieht. Die neuen Substanzen erlauben eine pathogenetisch sinnvolle Therapie - mit weniger Hypoglykämien und weniger Gewichtszunahme oder gar -abnahme. Derzeit befindet sich die Diabetologie im Stimmungshoch. Mal sehen, wie es weitergeht.

Literatur: www.springermedizin.de/info-diabetologie 


\section{editorial}

Literatur zum Beitrag „Kardiovaskuläre Effekte der Antidiabetika: Achterbahnfahrt mit Schreck und Jubel" von PD Dr. med. Ovidiu Alin Stirban

1. The ORIGIN Trial Investigators. N Engl J Med 2012;367:319-28.

2. Sciriza et al. N Engl J Med 2013;369:1317-26.

3. Green et al. N Engl J Med 2015;373;232-42.

4. White et al. N Engl J Med 2013;369:1327-35.

5. Zinman et al. N Engl J Med 2015;373:2117-28.

6. Neal et al. N Engl J Med 2017; doi: 10.1056/NEJMoa1611925.

7. Marso et al. N Engl J Med 2016;375:311-22.

8. Marso et al. N Engl J Med 2016;375:1834-44.

9. Pfeffer et al. N Engl J Med 2015;373:2247-57. 